\title{
Transparent Electrodes: A Review of the Use of Carbon-Based Nanomaterials
}

\author{
Edgar J. López-Naranjo, ${ }^{1}$ Luis J. González-Ortiz, ${ }^{2}$ Luis M. Apátiga, ${ }^{3}$ \\ Eric M. Rivera-Muñoz, ${ }^{3}$ and Alejandro Manzano-Ramírez ${ }^{4}$ \\ ${ }^{1}$ Departamento de Ingeniería de Proyectos, Centro Universitario de Ciencias Exactas e Ingenierías, \\ Universidad de Guadalajara, C.P. 44430, Guadalajara, JAL, Mexico \\ ${ }^{2}$ Departamento de Química, Centro Universitario de Ciencias Exactas e Ingenierías, \\ Universidad de Guadalajara, C.P. 44430, Guadalajara, JAL, Mexico \\ ${ }^{3}$ Centro de Física Aplicada y Tecnología Avanzada, UNAM, A.P. 1-1010, C.P. 76000, Querétaro, QRO, Mexico \\ ${ }^{4}$ CINVESTAV-IPN Unidad Querétaro, C.P. 76230, Santiago de Querétaro, QRO, Mexico
}

Correspondence should be addressed to Edgar J. López-Naranjo; edgar.lopezn@academicos.udg.mx

Received 23 June 2016; Accepted 1 September 2016

Academic Editor: Jianing An

Copyright (C) 2016 Edgar J. López-Naranjo et al. This is an open access article distributed under the Creative Commons Attribution License, which permits unrestricted use, distribution, and reproduction in any medium, provided the original work is properly cited.

Transparent conducting electrodes (TCE) are extensively applied in a great range of optoelectronic and photovoltaic equipment (e.g., solar cells, touch panels, and flexible devices). Carbon-based nanomaterials are considered as suitable replacements to substitute traditional materials to manufacture TCE due to their remarkable characteristics, for example, high optical transmittance and outstanding electrical properties. In comparison with traditional indium tin oxide electrodes, carbon-based electrodes show good mechanical properties, chemical stability, and low cost. Nevertheless, major issues related to the development of good quality manufacture methods to produce carbon-based nanomaterials have to be overcome to meet massive market requirements. Hence, the development of alternative TCE materials as well as appropriate large production techniques that meet the requirements of a proper sheet resistance along with a high optical transparency is a priority. Therefore, in this work, we summarize and discuss novel production and synthesis methods, chemical treatments, and hybrid materials developed to satisfy the worldwide request for carbon-based nanomaterials.

\section{Introduction}

Carbon-based nanomaterials [i.e., carbon nanotubes (CNT) and graphene] show excellent physical and electrical characteristics, including an elevated conductivity and mobility [1-3]. Due to their high optical transmittance this type of materials is particularly appealing to be used as transparent conducting electrodes (TCE) in photovoltaic and display applications. Up until now, ITO (indium tin oxide) is the most used TCE material in organic electronic devices. ITO is a transparent material that exhibits remarkable optoelectronic properties, high transmittance (90\%), and sheet resistance of $\sim 10 \Omega$ /sq. Nevertheless, it presents some major drawbacks, including high costs, scarcity of indium, and brittleness, making it impossible to adapt to future applications. Additionally, ITO presents a relatively high refraction index $(\sim 2.0)$ that could produce unwanted reflections if it is used on a substrate with lower refraction index [4-7]. As a consequence, the development of alternative TCE materials that fulfill the main requirements of a high optical transparency and a proper sheet resistance is a priority [8]. Graphene and CNT films represent suitable alternatives to substitute ITO and meet the required specifications to be used as TCE [9-11].

Therefore, the present review is intended to summarize and analyze the different synthesis and production methods, as well as the new carbon-based nanomaterials developed in order to satisfy the requirements for the fabrication of TCE. 


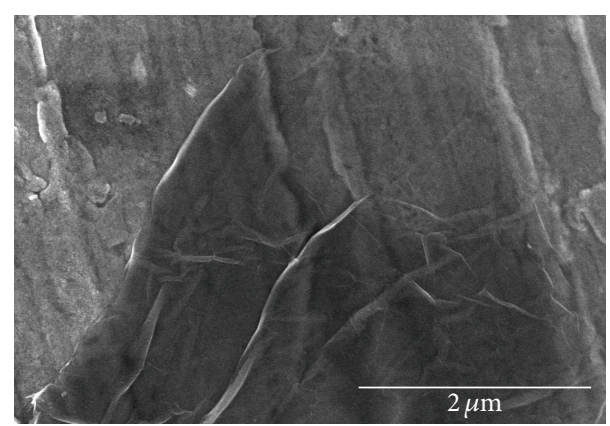

Figure 1: SEM micrograph of GO.

\section{Graphene and Graphene Derivatives}

Graphene, a carbon monolayer with two-dimensional honeycomb lattice structure, presents a great aspect ratio and outstanding properties (e.g., high optical transmittance, electrical conductivity and mechanical strength). Consequently, graphene seems to be a suitable alternative to replace oxidebased materials used as TCE in devices such as flexible displays, solar cells, and electronic equipment. However, massive use of graphene still faces major problems, for example, scale-up production and quality issues derived from manufacturing techniques, as well as inconveniences regarding transfer stage, which actually represents a major drawback in order to obtain an efficient electrode. To produce large-area graphene based TCE, the first goal is to generate enough high quality graphene sheets. However, it is well known that in this moment there is not a proper method to obtain abundant high-quality graphene. In addition, the use of graphene as an electrode demands an insulator substrate. Therefore, once graphene is synthesized, it is necessary to transfer it onto other proper substrates making the process more complex $[4,12-16]$.

Up until now, the most used technique to produce transparent conductive graphene films is CVD. In this method, a graphene film is commonly grown on a catalytic surface before being transferred to a final substrate in a separate procedure [17-19]. A critical stage in the study of the basic characteristics and practical uses of graphene includes such transfer procedure of graphene from a metal substrate to a desired target substrate without affecting the quality of the material [20]. Once graphene film is transferred onto a target substrate, it may be used as a transparent conducting electrode in a variety of flexible devices [21-23]. Other major inconveniences regarding the use of CVD-obtained graphene as transparent electrode are related to its high sheet resistance (compared to ITO-based electrodes), the difficulty to regulate the film thickness, and avoiding the generation of secondary crystals $[14,20,24,25]$.

Graphene oxide (GO), a graphene derivative, has also become an important material for the fabrication of TCE. GO (Figure 1) dispersions can be easily processed and deposited on different types of substrates to produce TCE $[26,27]$. To reinstate the intrinsic high electrical conductivity of graphene, GO needs to be reduced either in solution or after the films are formed on a particular substrate. Significant research has been performed regarding reduction techniques to restore $\mathrm{sp}^{2}$ carbon structure and consequently to increase electrical conductivity [28-30]. GO reduction techniques offer the possibility to obtain good-quality nanomaterials for TCE at low cost and appropriate for mass production [31]. Therefore, the use of GO constitutes a viable option to fabricate TCE.

Regarding the critical transfer stage of graphene films, various groups have proposed different and novel techniques to solve this issue. For example, Cai et al. [4] investigated a crackles transfer technique without the removal of the polymeric carrier, making it possible to successfully transfer graphene and give a place to a material with a sheet resistance of $219 \Omega /$ sq at $96.5 \%$ transmittance. In this work, graphene films were grown by a low-pressure CVD system on a transparent polymer used as substrate. Thus, such polymer did not need to be removed and the graphene film was not damaged during the process. Graphene film produced using this method showed a conductivity 5 times higher than that of undoped graphene films produced considering transfer with polymer removal. Chandrashekar et al. [32] also reported on the transfer of graphene films from $\mathrm{Cu}$ foil onto polymeric substrate by means of a delamination process to reduce the damage produced by the carrier elimination. Novel methods have also been proposed; for example, Bae et al. [33] reported on a novel roll to roll technique and wet chemical transfer doping of 30-inch graphene films grown by CVD onto copper substrates. The as-produced films showed sheet resistances as low as approximately $125 \Omega$ /sq with $97.4 \%$ optical transmittance and exhibited the half-integer quantum Hall effect, indicating their high quality.

With respect to large-scale fabrication, different techniques have been reported. For example electrochemical synthesis, which is known to be able to exfoliate graphene. By means of voltage control, the thickness, flake area, and the number of defects of the material can be manipulated [34]. Gee et al. [12] synthesized graphene through electrochemical exfoliation (EE) using graphite as starting material. Graphene was used to produce high quality bilayer films that showed a sheet resistance of $1.35 \times 10^{5} \Omega /$ sq with $70 \%$ light transmittance. Transparent films also proved to be bendable while maintaining electrical properties under deformation conditions. The resistance of the films could be considerably decreased by a posttreatment, such as thermal and/or chemical reduction. Kim et al. [35] described a technique to modify graphene via a simple, solvent-free, and efficient electrobeam irradiation using a CVD-prepared graphene. Results demonstrated that the graphene was successfully modified, resulting in an increase in its sheet resistance and work function. Graphene irradiated during $5 \mathrm{~s}$ exhibited the highest power conversion efficiency (i.e., 2.76\%), which was much better than that obtained when using nonirradiated graphene. Lee and $\mathrm{Ki}$ [36] reported on a simple and fully scalable laser-based method for fabricating large-scale, graphenebased transparent conductive films with customizable sheet resistance on glass substrates using amorphous carbon as 
the carbon source. In this method, a diamond-like carbon (DLC) was deposited on a glass substrate by pulsed laser deposition; then it was annealed in a helium shielding environment by a $2 \mathrm{~kW}$ continuous-wave fiber laser with a wavelength of $1070 \mathrm{~nm}$. DLC film, originally black, became transparent, and its transparency degree was found to depend on the process parameters. It was possible to obtain films with a sheet resistance value as low as $2050 \Omega /$ sq at $80 \%$ transparency from a $510 \mathrm{~nm}$ thick DLC film. Chen et al. [37] reported on a technique to obtain both transparent and stretchable graphene sheets for energy storage devices. The resultant wrinkled graphene sheets were able to be used as both current collector and electrode materials to build transparent and stretchable supercapacitors, which showed a high transparency $(57 \%$ at $550 \mathrm{~nm})$ and can be stretched up to $40 \%$ strain without obvious performance change over hundreds of stretching cycles.

As it was previously mentioned, reduction of GO offers the possibility to produce good-quality materials for TCE at low cost and suitable for mass production. Regarding this process, different routes have been reported, for example, thermal reduction of GO, which is a useful technique to produce graphene-based materials and devices [38]. Based on this technique, Kim et al. [39] presented a new procedure to obtain flexible transparent conductive graphene films; such a method consisted of two stages: a solution-processed chemical reduction and an anodized aluminum oxide (AAO) membrane filter-assisted thermal reduction. The final transparent graphene films produced by this technique showed a sheet resistance of less than $850 \Omega /$ sq with $80 \%$ transmittance under visible light irradiation (540 to $840 \mathrm{~nm}$ ). Sheet resistance was much lower than that of graphene films obtained from solution-processed approaches. Nguyen et al. [40] reported on an easy and nonharmful technique to obtain reduced graphene oxide (RGO) thin films on glass substrate at room temperature using a nontoxic solvent and RGO colloidal suspensions. RGO suspensions were deposited on simple and plasma treated glass substrates by drop casting. According to the results the transmittance and the conductivity of the films showed a linear relationship with the thickness of the deposited films. Plasma treatment was found to increase the conductivity when compared to the nontreated glass. Finally, Cote et al. [41] proved that it is possible to obtain stable monolayers of graphite oxide single layers without the need for any surfactant or stabilizing agent, due to the strong electrostatic repulsion between the 2D confined layers, which also prevented the single layers from overlapping during compression, leading to excellent reversibility of the monolayers. Monolayers were then transferred to a substrate, readily creating a large area of flat graphite oxide single layers. Graphite oxide monolayers could be chemically reduced to graphene for electronic applications such as transparent conducting films. Results indicated that the RGO films exhibited high electrical conductivities at both room and cryogenic temperatures.

Using spin coating, which is one of the most used techniques employed to deposit uniform films on a substrate [42], Yun et al. [43] investigated the morphological, optical, and electrical properties of films obtained using a
RGO solution. Spin-coated RGO thin films showed a highly uniform morphology with thickness controllability. Additionally, sheet resistance was efficiently decreased from $\sim 10^{3}$ to $10 \mathrm{k} \Omega / \mathrm{sq}$ by the combination of controlled coating cycles and annealing conditions while conserving the transmittance values between 57 and $87 \%$. Synthesized films were tested in organic photovoltaic cells; the performance of the devices was gradually increased as the sheet resistance decreased and the highest efficiency was of $0.33 \%$ and it was obtained using $200^{\circ} \mathrm{C}$-annealed and 7-times coated RGO film. Eda et al. [44] described an easy and reproducible technique to uniformly deposit between one and five layers of graphene from RGO in the form of thin films to create transistors and proofof-concept electrodes for organic photovoltaics. Since it was possible to obtain monolayer and multilayer (5 layers) materials using this technique, the optoelectronic properties could be modified over several orders of magnitude. Thicknesses of the produced films were found to vary from 1 to $5 \mathrm{~nm}$. Results showed that the thinnest films exhibited graphenelike ambipolar transistor characteristics, while thicker films showed graphite-like-semi-metals properties.

In addition, Kim et al. [45] built a transparent and flexible graphene charge-trap memory consisting of a single layer graphene channel and a 3-dimensional gate stack using a polyethylene naphtalate substrate. The device exhibited a transparency of $\sim 80 \%$ in the visible wavelength. Wang et al. [46] reported on the use of transparent, conductive, and ultrathin graphene films as electrodes for Gratzel solar cells. Exfoliated graphite oxide-based electrodes exhibited a high conductivity of $550 \mathrm{~S} / \mathrm{cm}$ and a transparency of $\sim 70 \%$ over $1000-3000 \mathrm{~nm}$ and remarkable chemical and thermal stabilities. And Liu et al. [47] reported on the use of a single-layer graphene film as the top electrode of semitransparent organic solar cells. The conductance of the graphene film was increased for more than $400 \%$ when it was doped with $\mathrm{Au}$ nanoparticles and poly(3,4-ethylenedioxythiophene):poly(styrenesulfonate) (PEDOT:PSS), doping also improved the performance of the photovoltaic device. Maximum efficiency was achieved in the devices with the area of $20 \mathrm{~mm}^{2}$ illuminated from graphene electrode under an AM 1.5 solar simulator. It is important to remark that all of the devices showed higher efficiency from the graphene than ITO side, which was attributed to the better transmittance of the graphene electrodes. It would be expectable that higher efficiencies could be reached using higher quality single-layer graphene and improved processing conditions.

Finally, the Langmuir-Blodgett (L-B) method, a sophisticated technique to manipulate the interfacial molecular orientation and packing, which allows obtaining tailored patterned structures on solid substrates [48] was also tested. Based on this technique, Zheng et al. [49] synthesized monolayer ultra-large graphene oxide (UL-GO) sheets with diameter up to about $100 \mu \mathrm{m}$ using a chemical method. Transparent conductive films were then produced using the UL-GO sheets that were deposited on a substrate by the L-B assembly technique, which is an easy, low cost, and tunable technique used for mass production. The films 
fabricated from UL-GO sheets with a closed-packed flat structure exhibited remarkable high electrical conductivity and transparency after thermal reduction. A sheet resistance of $605 \Omega /$ sq at $86 \%$ transparency was obtained, which is better than those of graphene films prepared by CVD.

2.1. Hybrid Materials. Hybrid materials constitute an important branch of materials science; this class of materials offers the possibility to take advantage of the properties of their components in order to produce composites with remarkable characteristics. Combining graphene with other type of materials such as polymers and other carbon-based nanomaterials has produced composites with better properties than traditional TCE materials. Ji et al. [50] prepared a highly concentrated graphene $(\mathrm{G})$ dispersion assisted by sulfonated carbon nanotubes (SCNT) to be used as transparent conductive electrode. The interconnected network conductive coating (G:SCNT) showed better transmittance and conductivity compared to commercial PET/ITO films. Additionally, it showed excellent mechanical flexibility and durability under bending cycles. Ma et al. [51] prepared a sugar derived carbon/graphene composite material by mixing glucose and graphene oxide using ultrasonication and calcination. The incorporation of sugar-derived carbon into graphene layers not only prevented the agglomeration of graphene sheets, but also improved electrolyte-electrode accessibility as well as electrode conductivity. This composite material exhibited a high specific capacitance $(273 \mathrm{~F} / \mathrm{g}$ at a current density of $0.5 \mathrm{~A} / \mathrm{g}$ ) as well as excellent electrochemical stability. Due to this remarkable performance, it is a promising material to be used as an efficient electrode based on environmentally friendly materials and low fabrication cost. Mahala et al. [52] reported on the design and characterization of solar cells using exfoliated graphene:PEDOT:PSS and PEDOT:PSS:RGO as transparent and current spreading electrode (TCSE). Results indicate that graphene/conducting polymer composites showed a great potential to be used as TCSE. Voronin et al. [53] demonstrated the possibility to create a stable hybrid coating based on the hybrid of a reduced graphene oxide (RGO)/Ag quasi-periodic mesh. The proposed method presents main advantages such as low cost of the processes and the technology scalability. The AG qmesh was formed via magnetron sputtering of silver, and the protective RGO film was formed by low temperature reduction of a graphene oxide film, applied by spray deposition in a solution of $\mathrm{NaBH}_{4}$. Coatings showed a low sheet resistance (12.3 $\Omega / \mathrm{sq})$, a high optical transparency $(82.2 \%)$, and a high chemical stability in addition to high stability to deformation impacts. Finally, Yu and Dai [54] reported the obtention of positively charged RGO sheets using hydrazine to reduce GO, and polyethyleneimine (PEI) as stabilizer. PEI-modified RGO films were sequentially self-assembled along with negatively charged acid-oxidized MWCNT. The resultant hybrid material could be used to fabricate largearea multicomponent films with a well-defined architecture and tunable thickness on different substrates, suitable for electrochemical applications.

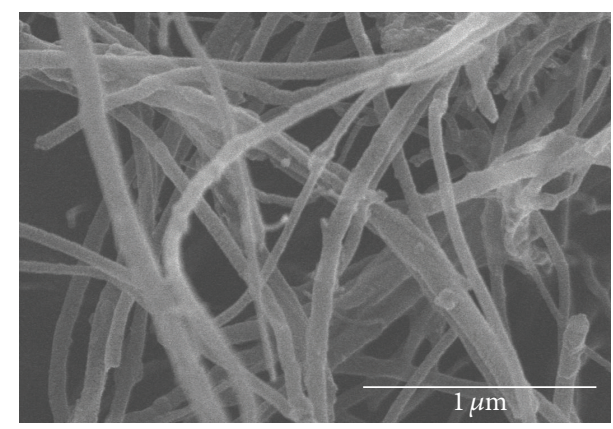

FIGURE 2: SEM micrograph of carbon nanotubes.

\section{Carbon Nanotubes}

Carbon nanotubes (Figure 2) can be described as sheets of graphene arranged in cylindrical shape and exist as both single-walled carbon nanotubes (SWCNT) and multiwalled carbon nanotubes (MWCNT) [66]. Due to their flexibility, high optical transparency, and conductivity, carbon nanotubes films have been considered an ideal candidate for applications in optoelectronics. The most important asset of CNT over ITO is their ability to be produced on flexible substrates. Although CNT show a great potential to substitute ITO in optoelectronic applications, there are still a lot of issues that need to be solved to allow the widespread use of this technology. At the present time, there are different options to fabricate flexible conductive electrodes based on CNT, such as direct deposition using spin coating, spraying or incubation, and deposition by vacuum filtration, as well as different indirect methods, including bar coating, dip coating, and inkjet printing. However, these methods show a major common problem, that is, the weak adhesion between the substrates and CNT [5, 67-71].

3.1. SWCNT. SWCNT electrodes present remarkable advantages compared to other typical materials used as electrodes. SWCNT show better electrochromic properties, a wider potential window than ITO, and are more resistant to acids. An important step towards a widespread use of SWCNT optically transparent electrodes is to develop easy techniques to produce stable electrodes with well-controlled properties. Therefore, different routes have been proposed in order to meet such aim [72, 73]. For example, Wu et al. [11] described a simple process for the production of ultrathin, transparent, optically homogenous, electrically conducting films of pure SWCNT and the transfer of those films to different substrates. For equivalent sheet resistance, the films exhibited optical transmittance comparable to that of commercial ITO in the visible spectrum, but far superior transmittance in the infrared spectral band. Garoz-Ruiz et al. [72] presented a simple method using a CVD reactor for the fabrication of optically transparent electrodes using SWCNT using organic solvents. Authors found that transparency and conductivity of the electrodes depended on the SWCNT concentration and filtered volume. Thus, to maximize such parameters it is extremely important to optimize fabrication conditions. 
Via spin coating Jo et al. [55] synthesized SWCNT using an oligothiophene-terminated poly(ethylene glycol) as nonionic amphiphilic surfactant. Due to the absence of charge repulsion between SWCNT/surfactant, a successful formation of a dense network of SWCNT was achieved. The sheet resistance of SWCNT was improved when treated with nitric acid and thionyl chloride after being cleaned with dichloromethane and water. Final sheet resistance was of $59 \Omega / \mathrm{sq}$, and a transparency of $71 \%$ at $550 \mathrm{~nm}$ was registered for the synthesized SWCNT.

Zhang et al. [56] prepared transparent conducting SWCNT films on both glass and polyethylene terephthalate (PET) using SWCNT dispersed by Nafion in water/ethanol with different ratios. It was found that films obtained at a 50:50 ratio exhibited the best performance, showing a sheet resistance of 500-600 $\Omega / \mathrm{sq}$ at transmittance of $85 \%$ and the best thermal stability at $60^{\circ} \mathrm{C}$ with change of sheet resistance less than $2 \%$ in $200 \mathrm{~h}$. The performance of such films was attributed to a small bundle size of SWCNT and the conductivity and thermal stability of Nafion. Jung et al. [74] produced a conductive thin film from a dispersion containing SWCNT, sodium dodecylbenzenesulfonate (NaDDBS), and a fluorosurfactant to reduce the surface tension, preventing agglomeration and improving the quality of the CNT films.

For some applications flexibility is preferred over transparency. Therefore, some research groups have focused on such feature. One way to achieve this goal is employing flexible substrates. Ferrer-Anglada et al. [57] prepared SWCNT transparent films using a flexible silicone as substrate by a simple spray method. Optical absorption and electrical conductivity were determined, for samples showing $65 \%$ to $85 \%$ optical transmittance and a sheet resistance of 1 to $8.5 \mathrm{k} \Omega / \mathrm{sq}$. When a thicker film of SWCNT was used, electrical resistance as low as $200 \Omega /$ sq was registered. Jung et al. [75] constructed mechanically flexible and optically transparent thin film solid state supercapacitors assembling nanoengineered carbon electrodes. Carbon films were transparent and showed a transmittance of $71 \%$ at $550 \mathrm{~nm}$, and the fabricated solid-state supercapacitor devices were also optically transparent and mechanically flexible. In addition, a simple and reproducible method to produce SWCNT optical transparent electrodes for optical transmission spectroelectrochemistry on flexible PET supports was reported by Heras et al. [73]. SWCNT were grown by aerosol chemical vapor deposition which can be considered as a dry, surfactant-assisted free alternative to vacuum filtration deposition. Using this technique, it was possible to prepare electrodes with different transparency and sheet resistance by simply modifying the collection time. Results indicated that the transparent electrodes showed a high transparency (up to $92 \%$ transmittance at $550 \mathrm{~nm}$ ) and excellent electrochemical properties.

Finally, Rowell et al. [58] fabricated transparent conducting electrodes by printing films of SWCNT networks on plastic using commercially available SWCNT produced by arc-discharge. It was demonstrated that it is possible to use SWCNT as transparent electrodes for efficient, flexible polymer-fullerene bulk heterojunction solar cells. Relatively smooth and homogeneous films presented a transmittance of $85 \%$ at $550 \mathrm{~nm}$ and a sheet resistance of $200 \Omega / \mathrm{sq}$. Cells were fabricated on the SWCNT/plastic anodes identically to a process optimized for ITO/glass. Results showed that it was possible to obtain efficiencies of $2.5 \%$, which are close to those using ITO/glass (3\%).

3.2. MWCNT. MWCNT, which are known to show excellent characteristics such as large surface area, high conductivity, and good chemical stability due to their structural properties, have also been used to produce TCE using different manufacturing techniques. For example, Aloui et al. [5] prepared flexible electrodes using PET as substrate. CNT were prepared in an ultrasonic bath using sodium dodecyl sulfate (SDS) aqueous solutions of carbon nanotubes. The performances of thin conductive films were evaluated using varying filtration concentrations. Their optimum result was achieved with a concentration of $1.2 \mathrm{mg} / \mathrm{mL}$; at this concentration, the sheet resistance was of $180 \Omega$ /sq with an optical transparency of about $81 \%$ at $550 \mathrm{~nm}$. The optoelectronic performance of carbon nanotubes depended on the preparation technique and on the $\mathrm{I}_{\mathrm{G}} / \mathrm{I}_{\mathrm{D}}$ ratio. Authors also obtained flexible conductive electrodes having the following structure: PET-MWCNTs/P3HT:PC70BM/Al to be used as organic diodes, and overall results demonstrated that it is possible to successfully integrate such electrodes in organic devices. Kim et al. [59] developed semitransparent n-i-p organic solar cells (OSC) with free-standing MWCNT sheets as transparent electrodes. OSC using MWCNT top anode electrodes showed very low leakage currents, high fill factors (up to $60 \%$ ) and promising efficiencies (up to $1.5 \%$ ), transparencies, and long-term stability. MWCNT sheets produced by CVD showed a sheet resistance $\sim 250 \Omega /$ sq and a transparency $\sim$ $38 \%$ (at $550 \mathrm{~nm}$ ). Grüner [9] obtained a two-dimensional transparent film based on carbon nanotubes with remarkable electrical, optical, mechanical, and environmental resistance properties suitable for a variety of emerging technologies ranging from macroelectronics to solid state lighting, organic solar cells, and smart fabrics. Williams et al. [76] used transparent carbon nanotube sheets as the hole-injecting anode of organic light emitting diodes. The as-fabricated devices showed a brightness of $4500 \mathrm{~cd} / \mathrm{m}^{2}$ and a current efficiency of $\sim 2.5 \mathrm{~cd} / \mathrm{A}$, which is similar to the efficiency of a device using ITO as the anode. According to the results, it was demonstrated that in order to achieve a high efficiency a proper planarization of the electrode using PEDOT:PSS is mandatory. Results also indicate that increasing conductivity of the carbon sheets could lead to CNT-based devices with efficiencies exceeding those of devices employing ITO.

3.3. Aligned Carbon Nanotubes. An aligned carbon nanotube array is a type of vertically aligned material that can be transformed into films, fibers, and yarns by means of different processes (e.g., extrusion, spinning, and twisting), showing unique mechanical, electrical, thermal, and electrochemical properties. Specifically, the synthesized films show high transparency and elevated conductivity. On the other hand, among the different types of CNT yarns, dry-spun materials have showed the best mechanical properties (i.e., high 
mechanical strength, tenacity, and resistance to abrasion) $[77,78]$.

Regarding its applications, it was first reported that twistless yarn drawn from carbon nanotube forest could be used as an incandescent lamp filament [79]. Due to its remarkable mechanical properties, many applications of CNT films and yarns, which take advantage of such features, have been explored so far (e.g., as materials with a high mechanical performance and as reinforcement in structured composites). Nevertheless, some researchers have demonstrated its use in the field of optoelectronic and photovoltaic devices since yarns present very appealing optical features such as high light transmission and good conductivity [78]. For example, Lima et al. [80] proved that the presence of CNT yarn optimizes light absorption when used as a substrate for $\mathrm{TiO}_{2}$.

Additionally, vertically aligned CNT (VACNT) forests have been tested as counter electrodes in Gratzel cells. Growing VACNT directly on the substrate is the most efficient technique since it produces a highly dense surface area of CNT directly attached to the substrate, showing an elevated electrical contact [81]. Anwar et al. [81] reported on the use of different substrates to growth VACTN to be used in Gratzel devices. One of the main drawbacks regarding the growth of VACTN are the high temperatures and severe atmospheres involved in the process, which represents a serious problem since the more commonly used substrates do not stand such hard processing conditions. Results indicated that aluminum and stainless steel could be used for CNT growth. However, the performance of the final device does not seem to be better than that of a traditional cell using Pt as counter electrode. In the same way, Nam et al. [82] reported on the use of aligned CNT as counter electrode in Gratzel cells, achieving efficiencies of over $10 \%$ due to a large and highly conductive surface area generated by wellaligned CNT. The CNT-deposited electrode showed higher photo-conversion efficiency when compared to traditional $\mathrm{Pt}$ counter electrodes.

Aligned CNT have also been used in composite materials for photovoltaic applications. For example, Choi et al. [83] employed a graphene-based MWCNT structure as counter electrode in a Gratzel device. Vertically grown MWCNT were synthesized onto graphene layers by CVD. According to electrochemical results, charge transfer using composites was higher than that registered for devices using single MWCNT or graphene electrode.

The future use of this type of materials depends on the ability to overcome current issues such as its scale-up to meet industrial demands and achieve size control [78].

3.4. Chemical Pretreatments. Pretreatment of CNT as well as surface modification processes to pretreat polymeric substrates can also modify the properties of CNT used as flexible transparent electrodes $[60,67,84]$, for example, corona pretreatment, a commercially useful technique of surface modification of plastic substrate that can be carried out at room conditions [68].

In this regard, Han et al. [67] reported the effect of coronatreated PET substrates on the properties of CNT deposited via spray coating on such polymer. According to their results, the surface roughness of the PET substrates was significantly increased as well as the surface energies. On the other hand, the contact angle increased and decreased depending on nature of the liquid used to run the test, indicating that the chemical properties of the substrate were changed. Park et al. [60] reported on the effects of sonificator and process time on the properties of CNT films obtained through spray coating using PET as substrate. Authors reported that films produced after ten cycles of spray coating presented the best performance showing a transmittance of $81 \%$ and a sheet resistance of $310 \Omega /$ sq. Additionally, Han et al. [68] studied the effects of the surface modification via corona treatment of PET substrates before the deposition of CNT varying the corona energies (from $149 \mathrm{~kJ} / \mathrm{m}^{2}$ to $5263 \mathrm{~kJ} / \mathrm{m}^{2}$ ), PET feeding directions, and number of corona treatments (1 to 4 ). Results based on the measurements of the changes in surface roughness, contact angles, and surface energies, indicated that as the number of treatments of the PET substrates increased, the water-based contact angles were found to be decreased, leading to an increase in the polar components of the surface energies, while diiodomethane-based contact angles increased, causing a decrement in the dispersive components of the surface energies, indicating the change in the chemical characteristics of the substrates and improving the adhesion of the CNT after corona pretreatment.

3.5. Carbon Nanosheets. Na et al. [85] demonstrated that pitch-converted carbon nanosheets (CNS) films can be used as TCE for OSC. A solution processed CNS film was prepared with spin coating using a pitch solution dissolved in dimethylformamide on quartz substrates, followed by stabilization and carbonization treatments to convert the pitch into CNS. CNS films were used as anode in OSC. Fabricated devices exhibited a high power conversion efficiency of $\sim 1.7 \%$ under $100 \mathrm{nW} \mathrm{cm}^{-2}$ illumination and AM $1.5 \mathrm{G}$ conditions. Results indicated that the thickness of CNS could be easily varied with the pitch concentration control.

3.6. Hybrid Materials. Graphene and CNT share an important number of structural and physical characteristics. Thus, it would be expected that their hybridization would give place to synergetic effects in optical and electrical properties [86]. Therefore, some groups have investigated on the properties of such hybrid films although until now very limited work has been reported. Souza et al. [61] prepared different types of thin films based on reduced graphene oxide and CNT using a liquid-liquid interfacial technique and deposited over two different substrates (i.e., glass and polyethylene terephthalate, PET). Three types of CNT were used without further purification: MWCNT with average diameter of $9.5 \mathrm{~nm}$ and average length of $1.5 \mu \mathrm{m}$, MWCNT with average diameter varying between 0.8 to $1.2 \mathrm{~nm}$ and average length between 100-1000 m, and MWCNT with average diameter of $40 \mathrm{~nm}$ and average length between 1-25 $\mu \mathrm{m}$. The reduced graphene oxide was synthesized by modified Hummers method. To evaluate the potential of the as-prepared films, 
sheet resistance, morphology, transmittance, and electrochemical measurements were analyzed. Results indicated that all transparent-flexible films could be successfully used as electrodes, presenting optimized sheet resistances ranging from 1.7 to $50 \mathrm{k} \Omega / \mathrm{sq}$ and transmittances at $550 \mathrm{~nm}$ up to $85 \%$. Yadav et al. [87] reported on a simple, facile, low cost effective vacuum filtration technique to obtain hybrid graphene/MWCNT films to be used as transparent electrodes for optoelectronic devices. Obtained films can be transferred on any substrate by placing the membrane into deionized water for self-releasing of the film from the filter membrane; such a transfer process has nearly yielded a 100\% effectiveness independent of the substrate. Graphene/WMCNT played an important role in synthesizing a transferrable film. The prepared films showed a transmittance up to $87.3 \pm 1 \%$ at $550 \mathrm{~nm}, 87.9 \pm 1 \%$ at $800 \mathrm{~nm}$ and sheet resistance of $136 \pm$ $22.4 \Omega /$ sq. Zhou et al. [62] proposed a technique to achieve an efficient and stable nanotube-nanotube interconnection to translate the remarkable properties of individual CNT to twodimensional networks using copper-halide crystallites. Crystallites worked as interconnecting modes when located at the cross points of CNT-CNT network. The presence of such dopant greatly improved the electrical conductivity of CNT films. Single-walled CNT were synthesized via enhanced direct injection pyrolytic synthesis method. Results indicated that CNT-halide hybrid films showed a sheet resistance of 5565 and $90-100 \Omega /$ sq at $85 \%$ and $90 \%$ optical transmittance at $550 \mathrm{~nm}$, respectively. Li et al. [63] fabricated composite thin films made of a carbon nanotube network patched with graphene sheets, assembled by a solid-phase layer-stacking approach with ethanol wetting. Composite films showed to be highly flexible, transparent, and conductive, with a sheet resistance of $735 \Omega / \mathrm{sq}$ at $90 \%$ transmittance at $550 \mathrm{~nm}$. Under AM 1.5 illumination, heterojuncton solar cells made from the composite films and n-type silicon showed a power conversion efficiency $>5.2 \%$. Battumur et al. [88] reported on a simple technique to produce MWCNT/graphene composites employing doctor-blade method and the performance of these hybrid materials used as electrode in dye-sensitized devices. The electrode composed of $60 \%$ MWCNT and $40 \%$ graphene showed the higher conversion efficiency (i.e., $4 \%)$ under illumination $(100 \mathrm{~mW} / \mathrm{cm})$, which is comparable registered using a Pt counter electrode.

Hybridization of graphene with other conducting materials such as metallic nanostructures, polymers, and grids allows the reduction of the structural defects of graphene without losing its high transparency [89]. In a general manner the use of polymers to produce carbon-based composites contributes to cost reduction. Specifically, the properties of each polymer contribute to modify the final properties of the composite, for example, polypyrrole, an organic polymer with good catalytic activity for $\mathrm{I}^{-3}$ reduction, high conductivity, and stability in the presence of liquid electrolytes. In their work, Gemeiner et al. [90] synthesized polypyrrole-coated-MWCNT structures via a threestep oxidative polymerization to be used as counter electrode in Gratzel devices. Compared to pristine polypyrrole electrodes, the presence of MWCNT improves charge transport, enhancing catalytic activity and consequently the performance of the final device. Other polymers such as polyaniline, poly methyl-methacrylate, polyvinylpyrrolidone, and PEDOT:PSS have also been tested. Ge et al. [91] produced transparent and flexible supercapacitors assembled from polyaniline (PANI)/SWCNT thin film electrodes. Ultrathin, optically homogeneous, transparent, and electrically conducting films showed a large specific capacitance due to combined double-layer capacitance and pseudo-capacitance mechanisms. Devices built using such electrodes gave a specific capacitance of $55.0 \mathrm{~F} / \mathrm{g}$ at a current density of $2.6 \mathrm{~A} / \mathrm{g}$, showing its viability for transparent and flexible. Jakubowska et al. [92] produced conductive and optically transparent poly methyl-methacrylate (PMMA) CNT films via screen printing to be used in the production of printed electronic paper. The obtained PMMA-CNT composites successfully sustained heavy mechanical stress or environmental factors such as periodical rapid temperature changes. Kim et al. [64] provided information regarding hybrid-type transparent electrodes fabricated depositing CNT on glass substrates via spray coating and then depositing thin PEDOT:PSS films on the CNT via spin coating that possess characteristics for touch screen panels. Experimental results showed CNT electrodes could satisfy the requirements necessary for touch screen panels such as a sheet resistance $100 \Omega$ /sq and visible transmittance higher than $80 \%$ and a neutral color (i.e., yellow approaching to zero). Yun et al. [89] reported on a highly efficient and bending durable all-carbon composite that employs uniform CNT networks on a monolayer graphene/polyethylene. Graphene was synthesized using a conventional CVD process. To keep the transparency and the flexibility of graphene, PET was introduced as substrate. CNT deposition was performed via spray deposition and spin coating. The aggregation of CNT was avoided using a heating temperature of $120^{\circ} \mathrm{C}$ during spray coating. It was possible to obtain a free-standing CNT/graphene assembly, making it possible to transfer the carbon-based material to a target substrate. The sheet resistance of the composite (CNT$4 \mathrm{~mL} /$ graphene/PET) was increased a $6 \%$ from its original value at a bending radius of $2.7 \mathrm{~mm}$, while that of the pristine graphene/PET increased $237 \%$. On the other hand, mechanical bending of the composite worsened the electrical performance by only $\sim 1.7 \%$ after 2000 bending cycles at a bending radius of $2.5 \mathrm{~mm}$. Kim et al. [65] fabricated hybridtype electrodes based on PEDOT:PSS:CNT via either spin coating or electrophoretic deposition (EPD) to improve the electric characteristics of CNT. To eliminate impurities in the CNT, CNT powder was placed in $\mathrm{H}_{2} \mathrm{SO}_{4}$ and $\mathrm{HNO}_{3}$. Purified CNT were filtered out using vacuum filtering and dispersed in an ultrasonic generator. CNT were deposited on glass via spray coating, and then PEDOT:PSS layers were deposited using spin coating and EPD. Thickness of the CNT increased from $40.5 \mathrm{~nm}$ to $112 \mathrm{~nm}$ by increasing the spraying time from 20 to $80 \mathrm{~s}$. On the other hand, as the spinning speed decreased, the thickness of the PEDOT:PSS films increased from $28.4 \mathrm{~nm}$ to $114.9 \mathrm{~nm}$. Hybrid electrodes showed a sheet resistance of $298 \Omega / \mathrm{sq}$ and a transmittance of $87.52 \%$. Park et al. [93] prepared SCNT wrapped with polyvinylpyrrolidone (PVP) to be used as counter electrode in Gratzel devices. 
TABLE 1: Sheet resistance of different carbon-based nanomaterials developed as potential substitutes for ITO.

\begin{tabular}{lccc}
\hline Material & Sheet resistance $(\Omega / s q)$ & Transparency (\%) & Reference \\
\hline Graphene & 219 & 96.5 & Cai et al. [4] \\
Graphene & 125 & 97.4 & Chandrashekar et al. [32] \\
Graphene & $1.35 \times 10^{5}$ & 70 & Gee et al. [12] \\
Graphene & 2050 & 80 & Lee and Ki [36] \\
Graphene & 850 & 80 & Kim et al. [39] \\
RGO & $10^{6}$ to $10^{3}$ & 57 to 87 & Yun et al. [43] \\
UL-GO & 605 & 86 & Zheng et al. [49] \\
RGO/Ag & 12.3 & 82.2 & Voronin et al. [53] \\
SWCNT & 59 & 71 & Jo et al. [55] \\
SWCNT & 500 to 600 & 85 & Zhang et al. [56] \\
SWCNT & 1000 to 85000 & 65 to 85 & Ferrer-Anglada et al. [57] \\
SWCNT & 200 & 85 & Rowell et al. [58] \\
MWCNT & 180 & 81 & Aloui et al. [5] \\
MWCNT & 250 & 38 & Kim et al. [59] \\
CNT & 310 & 81 & Park et al. [60] \\
RGO/MWCNT & 1700 to 50000 & 85 & Souza et al. [61] \\
CNT/copper halide & 55 to 65 & 85 to 90 & Zhou et al. [62] \\
CNT/graphene & 735 & 90 & Li et al. [63] \\
CNT/PEDOT:PSS & $<100$ & 80 & Kim et al. [64] \\
CNT/PEDOT:PSS & 298 & Kim et al. [65] &
\end{tabular}

Devices using composite materials showed efficiencies of $4.5 \%$, while those using pristine SCNT showed a conversion efficiency of $2.34 \%$, and the process for the obtention of SCNT composites allowed the decrease of the resistance of the electrode and to promote higher electrochemical reaction for better charge transfer reactions.

MWCNT have also been used with traditional ITO to produce hybrid materials. Ulbricht et al. [94] proved that strong transparent MWCNT play a main role as threedimensional hole-collecting network with extended interface connectivity to photoactive layer of bulk heterojunction organic photovoltaic devices. The obtained MWCNT showed a sheet resistance higher than that of ITO, and results indicated that incorporating MWCNT network to planar ITO, led to twice increased photocurrent. The performance of a hybrid anode device was found to be better $(\eta=1.74 \%)$ than those using a pure single layer of $\mathrm{CNT}(\eta=1.1 \%)$ or CNT:PEDOT:PSS $(\eta=1.43 \%)$.

As it is summarized in Table 1, different carbon-based nanomaterials showing a wide range of properties have been produced. In general, thin films of CNT or graphene are synthesized using different techniques such as spin coating [95, 96], spray coating $[97,98]$, dip coating $[46,99]$, LangmuirBlodgett deposition $[48,100]$, and chemical vapor deposition (CVD) $[18,101]$. Films synthesized using any of the previously mentioned methods need to be supported on a substrate and cannot be easily transferred to a different layer [87]. Although substrate-free films of CNT and graphene are also available, they present drawbacks in terms of transparency and conductivity $[63,102,103]$. Overcoming this type of issues along with problems related to massive production will help to increase the use of carbon-based nanomaterials as TCE. Different pretreatments and modifications to actual synthesis techniques as well as the development of new hybrid materials are being tested in order to find a solution to this problematic issue.

\section{Conclusions}

Due to the same basic structure, carbon-based nanomaterials (i.e., graphene and CNT) present similar characteristics such as remarkable optical and transport properties. Up until now, carbon-based nanomaterials constitute important potential alternatives to substitute traditional materials in the fabrication of transparent films. Although in some cases TCE made of carbon nanomaterials have outperformed those made of traditional ITO, there is still a large room for improvements to overcome the major issues that prevent massive use of carbon-based nanomaterials as TCE in modern optoelectronic devices.

\section{Competing Interests}

The authors declare that they have no competing interests.

\section{Acknowledgments}

The authors want to thank to Carmen Peza Ledesma for her technical support in electron microscopy analysis.

\section{References}

[1] A. K. Geim and K. S. Novoselov, "The rise of graphene," Nature Materials, vol. 6, no. 3, pp. 183-191, 2007. 
[2] K. S. Novoselov, A. K. Geim, S. V. Morozov et al., "Electric field effect in atomically thin carbon films," Science, vol. 306, no. 5696, pp. 666-669, 2004.

[3] P. Avouris, Z. Chen, and V. Perebeinos, "Carbon-based electronics," Nature Nanotechnology, vol. 2, no. 10, pp. 605-615, 2007.

[4] C. Cai, F. Jia, A. Li et al., "Crackless transfer of large-area graphene films for superior-performance transparent electrodes," Carbon, vol. 98, pp. 457-462, 2016.

[5] W. Aloui, A. Ltaief, and A. Bouazizi, "Transparent and conductive multi walled carbon nanotubes flexible electrodes for optoelectronic applications," Superlattices and Microstructures, vol. 64, pp. 581-589, 2013.

[6] G. U. Kulkarni, S. Kiruthika, R. Gupta, and K. D. M. Rao, "Towards low cost materials and methods for transparent electrodes," Current Opinion in Chemical Engineering, vol. 8, pp. 60-68, 2015.

[7] D. S. Hecht, L. Hu, and G. Irvin, "Emerging transparent electrodes based on thin films of carbon nanotubes, graphene, and metallic nanostructures," Advanced Materials, vol. 23, no. 13, pp. 1482-1513, 2011.

[8] A. Kasry, M. El Ashry, R. A. Nistor et al., "High performance metal microstructure for carbon-based transparent conducting electrodes," Thin Solid Films, vol. 520, no. 15, pp. 4827-4830, 2012.

[9] G. Grüner, "Carbon nanotube films for transparent and plastic electronics," Journal of Materials Chemistry, vol. 16, no. 35, pp. 3533-3539, 2006.

[10] J. L. Blackburn, T. M. Barnes, M. C. Beard et al., "Transparent conductive single-walled carbon nanotube networks with precisely tunable ratios of semiconducting and metallic nanotubes," ACS Nano, vol. 2, no. 6, pp. 1266-1274, 2008.

[11] Z. Wu, Z. Chen, X. Du et al., "Transparent, conductive carbon nanotube films," Science, vol. 305, no. 5688, pp. 1273-1276, 2004.

[12] C.-M. Gee, C.-C. Tseng, F.-Y. Wu et al., "Flexible transparent electrodes made of electrochemically exfoliated graphene sheets from low-cost graphite pieces," Displays, vol. 34, no. 4, pp. 315319, 2013.

[13] S. Pang, Y. Hernandez, X. Feng, and K. Müllen, "Graphene as transparent electrode material for organic electronics," Advanced Materials, vol. 23, no. 25, pp. 2779-2795, 2011.

[14] M. J. Allen, V. C. Tung, and R. B. Kaner, "Honeycomb carbon: a review of graphene," Chemical Reviews, vol. 110, no. 1, pp. 132$145,2010$.

[15] K. Rana, J. Singh, and J.-H. Ahn, "A graphene-based transparent electrode for use in flexible optoelectronic devices," Journal of Materials Chemistry C, vol. 2, no. 15, pp. 2646-2656, 2014.

[16] D. Konatham and A. Striolo, "Molecular design of stable graphene nanosheets dispersions," Nano Letters, vol. 8, no. 12, pp. 4630-4641, 2008.

[17] X. Li, W. Cai, J. An et al., "Large-area synthesis of high-quality and uniform graphene films on copper foils," Science, vol. 324, no. 5932, pp. 1312-1314, 2009.

[18] K. S. Kim, Y. Zhao, H. Jang et al., "Large-scale pattern growth of graphene films for stretchable transparent electrodes," Nature, vol. 457, no. 7230, pp. 706-710, 2009.

[19] W. Cai, Y. Zhu, X. Li, R. D. Piner, and R. S. Ruoff, "Large area few-layer graphene/graphite films as transparent thin conducting electrodes," Applied Physics Letters, vol. 95, no. 12, Article ID 123115, 2009.

[20] X. Li, Y. Zhu, W. Cai et al., "Transfer of large-area graphene films for high-performance transparent conductive electrodes," Nano Letters, vol. 9, no. 12, pp. 4359-4363, 2009.
[21] X. Wan, G. Long, L. Huang, and Y. Chen, "Graphene-a promising material for organic photovoltaic cells," Advanced Materials, vol. 23, no. 45, pp. 5342-5358, 2011.

[22] D. Jariwala, V. K. Sangwan, L. J. Lauhon, T. J. Marks, and M. C. Hersam, "Carbon nanomaterials for electronics, optoelectronics, photovoltaics, and sensing," Chemical Society Reviews, vol. 42, no. 7, pp. 2824-2860, 2013.

[23] X. Huang, Z. Zeng, Z. Fan, J. Liu, and H. Zhang, "Graphenebased electrodes," Advanced Materials, vol. 24, no. 45, pp. 59796004, 2012.

[24] J.-K. Choi, J. Kwak, S.-D. Park et al., "Growth of wrinkle-free graphene on texture-controlled platinum films and thermalassisted transfer of large-scale patterned graphene," ACS Nano, vol. 9, no. 1, pp. 679-686, 2015.

[25] V. Babenko, A. T. Murdock, A. A. Koós et al., "Rapid epitaxyfree graphene synthesis on silicidated polycrystalline platinum," Nature Communications, vol. 6, article 7536, 2015.

[26] Y. Zhu, S. Murali, W. Cai et al., "Graphene and graphene oxide: synthesis, properties, and applications," Advanced Materials, vol. 22, no. 35, pp. 3906-3924, 2010.

[27] D. R. Dreyer, S. Park, C. W. Bielawski, and R. S. Ruoff, "The chemistry of graphene oxide," Chemical Society Reviews, vol. 39, no. 1, pp. 228-240, 2010.

[28] I. Jung, D. Dikin, S. Park, W. Cai, S. L. Mielke, and R. S. Ruoff, "Effect of water vapor on electrical properties of individual reduced graphene oxide sheets," The Journal of Physical Chemistry C, vol. 112, no. 51, pp. 20264-20268, 2008.

[29] I. Jung, D. A. Dikin, R. D. Piner, and R. S. Ruoff, “Tunable electrical conductivity of individual graphene oxide sheets reduced at low temperatures," Nano Letters, vol. 8, no. 12, pp. 4283-4287, 2008.

[30] C. Mattevi, G. Eda, S. Agnoli et al., "Evolution of electrical, chemical, and structural properties of transparent and conducting chemically derived graphene thin films," Advanced Functional Materials, vol. 19, no. 16, pp. 2577-2583, 2009.

[31] S. Stankovich, D. A. Dikin, R. D. Piner et al., "Synthesis of graphene-based nanosheets via chemical reduction of exfoliated graphite oxide," Carbon, vol. 45, no. 7, pp. 1558-1565, 2007.

[32] B. N. Chandrashekar, B. Deng, A. S. Smitha et al., "Roll-to-roll green transfer of CVD graphene onto plastic for a transparent and flexible triboelectric nanogenerator," Advanced Materials, vol. 27, no. 35, pp. 5210-5216, 2015.

[33] S. Bae, H. Kim, Y. Lee et al., "Roll-to-roll production of 30-inch graphene films for transparent electrodes," Nature Nanotechnology, vol. 5, no. 8, pp. 574-578, 2010.

[34] M. Hofmann, W.-Y. Chiang, T. D. Nguyn, and Y.-P. Hsieh, "Controlling the properties of graphene produced by electrochemical exfoliation," Nanotechnology, vol. 26, no. 33, Article ID 335607, 2015.

[35] S.-H. Kim, Y.-J. Noh, S.-N. Kwon et al., "Efficient modification of transparent graphene electrodes by electron beam irradiation for organic solar cells," Journal of Industrial and Engineering Chemistry, vol. 26, pp. 210-213, 2015.

[36] K. Lee and H. Ki, "Rapid fabrication of transparent conductive films with controllable sheet resistance on glass substrates by laser annealing of diamond-like carbon films," Acta Materialia, vol. 111, pp. 315-320, 2016.

[37] T. Chen, Y. Xue, A. K. Roy, and L. Dai, "Transparent and stretchable high-performance supercapacitors based on wrinkled graphene electrodes," ACS Nano, vol. 8, no. 1, pp. 10391046, 2014. 
[38] Y. Qiu, F. Guo, R. Hurt, and I. Külaots, "Explosive thermal reduction of graphene oxide-based materials: mechanism and safety implications," Carbon, vol. 72, pp. 215-223, 2014.

[39] S. H. Kim, Y. Yu, Y. Z. Li, T. Xu, and J. F. Zhi, "A hybrid reduction procedure for preparing flexible transparent graphene films with improved electrical properties," Journal of Materials Chemistry, vol. 22, no. 35, pp. 18306-18313, 2012.

[40] C. V. Nguyen, R. Bartali, L. Crema, and G. Speranza, "Effect of glass surface treatments on the deposition of highly transparent reduced graphene oxide films by dropcasting method," Colloids and Surfaces A: Physicochemical and Engineering Aspects, vol. 498, pp. 231-238, 2016.

[41] L. J. Cote, F. Kim, and J. Huang, "Langmuir-blodgett assembly of graphite oxide single layers," Journal of the American Chemical Society, vol. 131, no. 3, pp. 1043-1049, 2009.

[42] H. A. Becerril, J. Mao, Z. Liu, R. M. Stoltenberg, Z. Bao, and Y. Chen, "Evaluation of solution-processed reduced graphene oxide films as transparent conductors," ACS Nano, vol. 2, no. 3, pp. 463-470, 2008.

[43] J.-M. Yun, C.-H. Jung, Y.-J. Noh et al., "Morphological, optical, and electrical investigations of solution-processed reduced graphene oxide and its application to transparent electrodes in organic solar cells," Journal of Industrial and Engineering Chemistry, vol. 21, pp. 877-883, 2015.

[44] G. Eda, G. Fanchini, and M. Chhowalla, "Large-area ultrathin films of reduced graphene oxide as a transparent and flexible electronic material," Nature Nanotechnology, vol. 3, no. 5, pp. 270-274, 2008.

[45] S. M. Kim, E. B. Song, S. Lee et al., "Transparent and flexible graphene charge-trap memory," ACS Nano, vol. 6, no. 9, pp. 7879-7884, 2012.

[46] X. Wang, L. Zhi, and K. Müllen, "Transparent, conductive graphene electrodes for dye-sensitized solar cells," Nano Letters, vol. 8, no. 1, pp. 323-327, 2008.

[47] Z. Liu, J. Li, Z.-H. Sun, G. Tai, S.-P. Lau, and F. Yan, “The application of highly doped single-layer graphene as the top electrodes of semitransparent organic solar cells," ACS Nano, vol. 6, no. 1, pp. 810-818, 2012.

[48] X. Li, G. Zhang, X. Bai et al., "Highly conducting graphene sheets and Langmuir-Blodgett films," Nature Nanotechnology, vol. 3, no. 9, pp. 538-542, 2008.

[49] Q.-B. Zheng, L.-F. Shi, and J.-H. Yang, "Langmuir-Blodgett assembly of ultra-large graphene oxide films for transparent electrodes," Transactions of Nonferrous Metals Society of China, vol. 22, no. 10, pp. 2504-2511, 2012.

[50] T. Ji, L. Tan, J. Bai, X. Hu, S. Xiao, and Y. Chen, "Synergistic dispersible graphene: sulfonated carbon nanotubes integrated with PEDOT for large-scale transparent conductive electrodes," Carbon, vol. 98, pp. 15-23, 2016.

[51] J. Ma, T. Xue, and X. Qin, "Sugar-derived carbon/graphene composite materials as electrodes for supercapacitors," Electrochimica Acta, vol. 115, pp. 566-572, 2014.

[52] P. Mahala, A. Kumar, S. Nayak, S. Behura, C. Dhanavantri, and O. Jani, "Graphene, conducting polymer and their composites as transparent and current spreading electrode in GaN solar cells," Superlattices and Microstructures, vol. 92, pp. 366-373, 2016.

[53] A. S. Voronin, F. Ivanchenko, M. Simunin et al., "High performance hybrid $\mathrm{rGO} / \mathrm{Ag}$ quasi-periodic mesh transparent electrodes for flexible electrochromic devices," Applied Surface Science, vol. 364, pp. 931-937, 2016.
[54] D. Yu and L. Dai, "Self-assembled graphene/carbon nanotube hybrid films for supercapacitors," Journal of Physical Chemistry Letters, vol. 1, no. 2, pp. 467-470, 2010.

[55] J. W. Jo, J. W. Jung, J. U. Lee, and W. H. Jo, "Fabrication of highly conductive and transparent thin films from single-walled carbon nanotubes using a new non-ionic surfactant via spin coating," ACS Nano, vol. 4, no. 9, pp. 5382-5388, 2010.

[56] J. Zhang, L. Gao, J. Sun et al., "Dispersion of single-walled carbon nanotubes by nafion in water/ethanol for preparing transparent conducting films," The Journal of Physical Chemistry C, vol. 112, no. 42, pp. 16370-16376, 2008.

[57] N. Ferrer-Anglada, J. Pérez-Puigdemont, J. Figueras, M. Z. Iqbal, and S. Roth, "Flexible, transparent electrodes using carbon nanotubes," Nanoscale Research Letters, vol. 7, no. 1, pp. 571-578, 2012.

[58] M. W. Rowell, M. A. Topinka, M. D. McGehee et al., "Organic solar cells with carbon nanotube network electrodes," Applied Physics Letters, vol. 88, no. 23, Article ID 233506, 2006.

[59] Y. H. Kim, L. Müller-Meskamp, A. A. Zakhidov et al., "Semitransparent small molecule organic solar cells with laminated free-standing carbon nanotube top electrodes," Solar Energy Materials and Solar Cells, vol. 96, no. 1, pp. 244-250, 2012.

[60] C. Park, S. W. Kim, Y.-S. Lee, S. H. Lee, K. H. Song, and L. S. Park, "Spray coating of carbon nanotube on polyethylene terephthalate film for touch panel application," Journal of Nanoscience and Nanotechnology, vol. 12, no. 7, pp. 5351-5356, 2012.

[61] V. H. R. Souza, S. Husmann, E. Neiva et al., "Flexible, transparent and thin films of carbon nanomaterials as electrodes for electrochemical applications," Electrochimica Acta, vol. 197, pp. 200-209, 2016.

[62] Y. Zhou, S. Shimada, T. Saito, and R. Azumi, "Building interconnects in carbon nanotube networks with metal halides for transparent electrodes," Carbon, vol. 87, pp. 61-69, 2016.

[63] C. Li, Z. Li, H. Zhu et al., "Graphene nano-'patches' on a carbon nanotube network for highly transparent/conductive thin film applications," The Journal of Physical Chemistry C, vol. 114, no. 33, pp. 14008-14012, 2010.

[64] B.-J. Kim, S.-H. Han, and J.-S. Park, "Sheet resistance, transmittance, and chromatic property of CNTs coated with PEDOT:PSS films for transparent electrodes of touch screen panels," Thin Solid Films, vol. 572, pp. 68-72, 2014.

[65] B.-J. Kim, S.-H. Han, and J.-S. Park, "Properties of CNTs coated by PEDOT: PSS films via spin-coating and electrophoretic deposition methods for flexible transparent electrodes," Surface and Coatings Technology, vol. 271, pp. 22-26, 2015.

[66] L. Hu, D. S. Hecht, and G. Grüner, "Carbon nanotube thin films: fabrication, properties, and applications," Chemical Reviews, vol. 110, no. 10, pp. 5790-5844, 2010.

[67] S.-H. Han, B.-J. Kim, and J.-S. Park, "Effects of the corona pretreatment of PET substrates on the properties of flexible transparent CNT electrodes," Thin Solid Films, vol. 572, pp. 7378, 2014.

[68] S.-H. Han, B.-J. Kim, and J.-S. Park, "Surface modification of plastic substrates via corona-pretreatment and its effects on the properties of carbon nanotubes for use of flexible transparent electrodes," Surface and Coatings Technology, vol. 271, pp. 100105, 2015.

[69] T. M. Barnes, J. D. Bergeson, R. C. Tenent et al., "Carbon nanotube network electrodes enabling efficient organic solar cells without a hole transport layer," Applied Physics Letters, vol. 96, no. 24, Article ID 243309, 2010. 
[70] V. Scardaci, R. Coull, and J. N. Coleman, "Very thin transparent, conductive carbon nanotube films on flexible substrates," Applied Physics Letters, vol. 97, no. 2, Article ID 023114, 2010.

[71] A. Schindler, J. Brill, N. Fruehauf, J. P. Novak, and Z. Yaniv, "Solution-deposited carbon nanotube layers for flexible display applications," Physica E: Low-Dimensional Systems and Nanostructures, vol. 37, no. 1-2, pp. 119-123, 2007.

[72] J. Garoz-Ruiz, S. Palmero, D. Ibañez, A. Heras, and A. Colina, "Press-transfer optically transparent electrodes fabricated from commercial single-walled carbon nanotubes," Electrochemistry Communications, vol. 25, pp. 1-4, 2012.

[73] A. Heras, A. Colina, J. López-Palacios et al., "Flexible optically transparent single-walled carbon nanotube electrodes for UVVis absorption spectroelectrochemistry," Electrochemistry Communications, vol. 11, no. 2, pp. 442-445, 2009.

[74] H. Jung, J. S. Yu, H. P. Lee, J. M. Kim, J. Y. Park, and D. Kim, "A scalable fabrication of highly transparent and conductive thin films using fluorosurfactant-assisted single-walled carbon nanotube dispersions," Carbon, vol. 52, pp. 259-266, 2013.

[75] H. Y. Jung, M. B. Karimi, M. G. Hahm, P. M. Ajayan, and Y. J. Jung, "Transparent, flexible supercapacitors from nanoengineered carbon films," Scientific Reports, vol. 2, article 773, 2012.

[76] C. D. Williams, R. O. Robles, M. Zhang, S. Li, R. H. Baughman, and A. A. Zakhidov, "Multiwalled carbon nanotube sheets as transparent electrodes in high brightness organic light-emitting diodes," Applied Physics Letters, vol. 93, no. 18, Article ID 183506, 2008.

[77] M. Miao, "Yarn spun from carbon nanotube forests: production, structure, properties and applications," Particuology, vol. 11, no. 4, pp. 378-393, 2013.

[78] K. Jiang, J. Wang, Q. Li, L. Liu, C. Liu, and S. Fan, "Superaligned carbon nanotube arrays, films, and yarns: a road to applications," Advanced Materials, vol. 23, no. 9, pp. 1154-1161, 2011.

[79] K. Jiang, Q. Li, and S. Fan, "Spinning continuous carbon nanotube yarns," Nature, vol. 419, no. 6909, article 801, 2002.

[80] M. D. Lima, S. Fang, X. Lepró et al., "Biscrolling nanotube sheets and functional guests into yarns," Science, vol. 331, no. 6013, pp. 51-55, 2011.

[81] H. Anwar, A. E. George, and I. G. Hill, "Vertically-aligned carbon nanotube counter electrodes for dye-sensitized solar cells," Solar Energy, vol. 88, pp. 129-136, 2013.

[82] J. G. Nam, Y. J. Park, B. S. Kim, and J. S. Lee, "Enhancement of the efficiency of dye-sensitized solar cell by utilizing carbon nanotube counter electrode," Scripta Materialia, vol. 62, no. 3, pp. 148-150, 2010.

[83] H. Choi, H. Kim, S. Hwang, W. Choi, and M. Jeon, "Dyesensitized solar cells using graphene-based carbon nano composite as counter electrode," Solar Energy Materials and Solar Cells, vol. 95, no. 1, pp. 323-325, 2011.

[84] H.-Z. Geng, K. K. Ki, P. S. Kang, S. L. Young, Y. Chang, and H. L. Young, "Effect of acid treatment on carbon nanotube-based flexible transparent conducting films," Journal of the American Chemical Society, vol. 129, no. 25, pp. 7758-7759, 2007.

[85] S.-I. Na, J.-S. Lee, Y.-J. Noh et al., "Efficient ITO-free polymer solar cells with pitch-converted carbon nanosheets as novel solution-processable transparent electrodes," Solar Energy Materials and Solar Cells, vol. 115, pp. 1-6, 2013.

[86] V. C. Tung, L.-M. Chen, M. J. Allen et al., "Low-temperature solution processing of graphene-carbon nanotube hybrid materials for high-performance transparent conductors," Nano Letters, vol. 9, no. 5, pp. 1949-1955, 2009.
[87] S. Yadav, V. Kumar, S. Arora, S. Singh, D. Bhatnagar, and I. Kaur, "Fabrication of ultrathin, free-standing, transparent and conductive graphene/multiwalled carbon nanotube film with superior optoelectronic properties," Thin Solid Films, vol. 595, pp. 193-199, 2015.

[88] T. Battumur, S. H. Mujawar, Q. T. Truong et al., "Graphene/carbon nanotubes composites as a counter electrode for dye-sensitized solar cells," Current Applied Physics, vol. 12, no. 1, pp. e49-e53, 2012.

[89] H. D. Yun, J. Kwak, S. Kim et al., "High performance all-carbon composite transparent electrodes containing uniform carbon nanotube networks," Journal of Alloys and Compounds, vol. 675, pp. 37-45, 2016.

[90] P. Gemeiner, J. Kuliček, M. Mikula et al., "Polypyrrole-coated multi-walled carbon nanotubes for the simple preparation of counter electrodes in dye-sensitized solar cells," Synthetic Metals, vol. 210, pp. 323-331, 2015.

[91] J. Ge, G. Cheng, and L. Chen, "Transparent and flexible electrodes and supercapacitors using polyaniline/single-walled carbon nanotube composite thin films," Nanoscale, vol. 3, no. 8 , pp. 3084-3088, 2011.

[92] M. Jakubowska, M. Słoma, and A. Młoniak, "Printed transparent electrodes containing carbon nanotubes for elastic circuits applications with enhanced electrical durability under severe conditions," Materials Science and Engineering B, vol. 176, no. 4, pp. 358-362, 2011.

[93] J.-G. Park, M. S. Akhtar, Z. Y. Li, D.-S. Cho, W. Lee, and O.-B. Yang, "Application of single walled carbon nanotubes as counter electrode for dye sensitized solar cells," Electrochimica Acta, vol. 85, pp. 600-604, 2012.

[94] R. Ulbricht, S. B. Lee, X. Jiang et al., "Transparent carbon nanotube sheets as 3-D charge collectors in organic solar cells," Solar Energy Materials and Solar Cells, vol. 91, no. 5, pp. 416-419, 2007.

[95] V. C. Tung, M. J. Allen, Y. Yang, and R. B. Kaner, "Highthroughput solution processing of large-scale graphene," Nature Nanotechnology, vol. 4, no. 1, pp. 25-29, 2009.

[96] D. Zhang, K. Ryu, X. Liu et al., "Transparent, conductive, and flexible carbon nanotube films and their application in organic light-emitting diodes," Nano Letters, vol. 6, no. 9, pp. 1880-1886, 2006.

[97] V. H. Pham, T. V. Cuong, S. H. Hur et al., "Fast and simple fabrication of a large transparent chemically-converted graphene film by spray-coating," Carbon, vol. 48, no. 7, pp. 1945-1951, 2010.

[98] E. Ramasamy, W. J. Lee, D. Y. Lee, and J. S. Song, "Spray coated multi-wall carbon nanotube counter electrode for tri-iodide $\left(I_{3}^{-}\right)$reduction in dye-sensitized solar cells," Electrochemistry Communications, vol. 10, no. 7, pp. 1087-1089, 2008.

[99] Y. Murakami, S. Chiashi, Y. Miyauchi et al., "Growth of vertically aligned single-walled carbon nanotube films on quartz substrates and their optical anisotropy," Chemical Physics Letters, vol. 385, no. 3-4, pp. 298-303, 2004.

[100] L. Feng, H. Li, F. Li, Z. Shi, and Z. Gu, "Functionalization of carbon nanotubes with amphiphilic molecules and their Langmuir-Blodgett films," Carbon, vol. 41, no. 12, pp. 2385-2391, 2003.

[101] C. Singh, M. S. P. Shaffer, and A. H. Windle, "Production of controlled architectures of aligned carbon nanotubes by an injection chemical vapour deposition method," Carbon, vol. 41, no. 2, pp. 359-368, 2003. 
[102] Z. Shi, X. Chen, X. Wang, T. Zhang, and J. Jin, "Fabrication of superstrong ultrathin free-standing single-walled carbon nanotube films via a wet process," Advanced Functional Materials, vol. 21, no. 22, pp. 4358-4363, 2011.

[103] J. C. Chen, Y. Guo, L. Huang et al., "Controllable fabrication of ultrathin free-standing graphene films," Philosophical Transactions of the Royal Society A: Mathematical, Physical and Engineering Sciences, vol. 372, no. 2013, Article ID 20130017, 2014. 

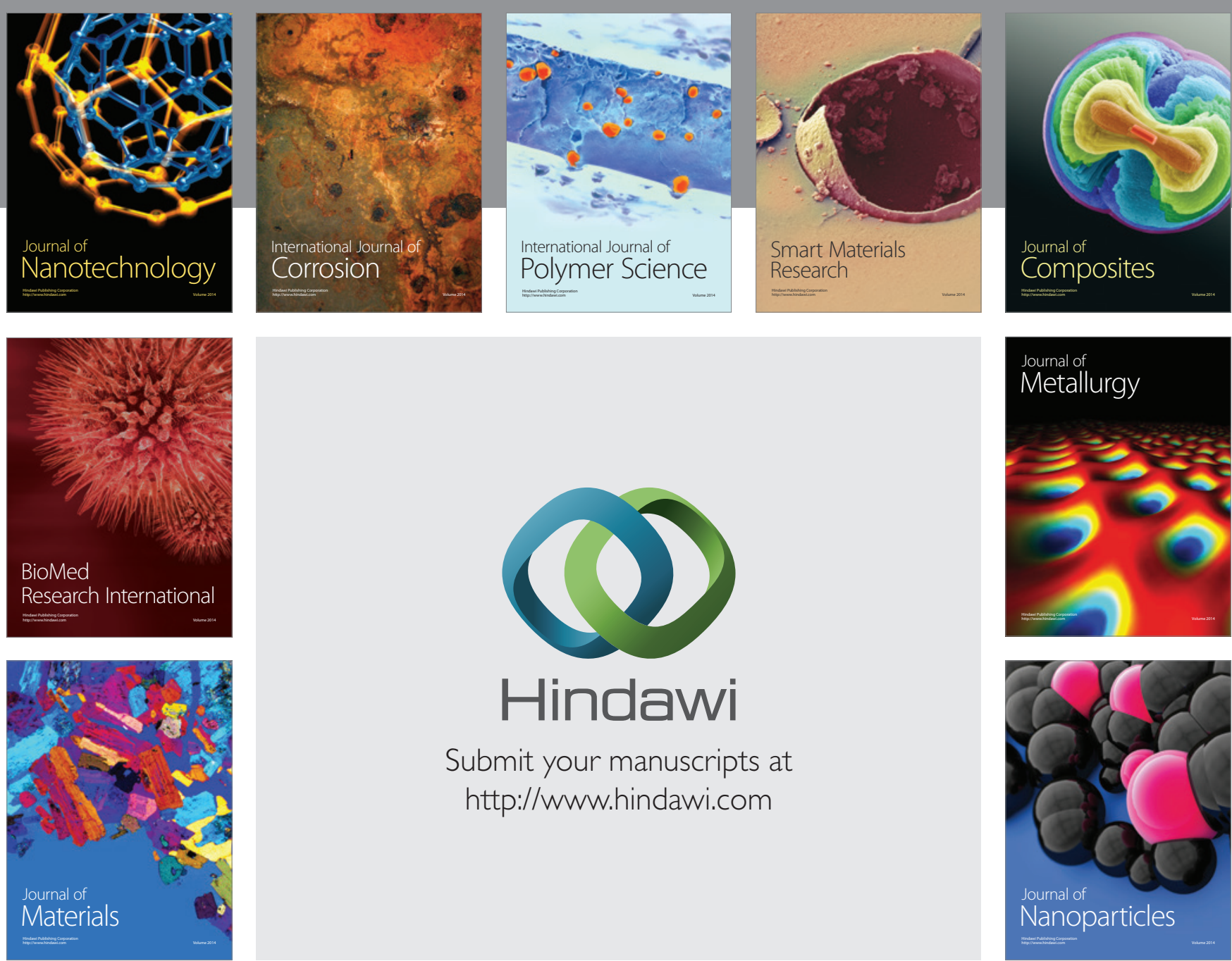

\section{Hindawi}

Submit your manuscripts at

http://www.hindawi.com

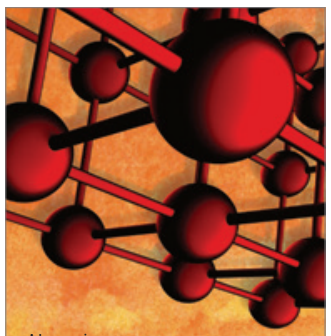

Materials Science and Engineering
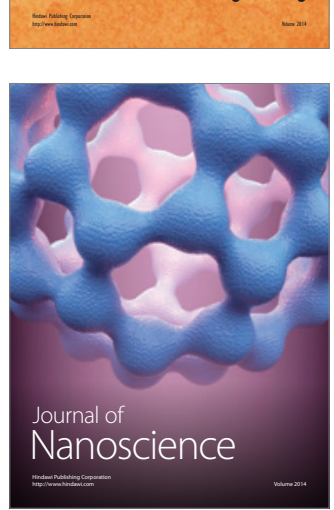
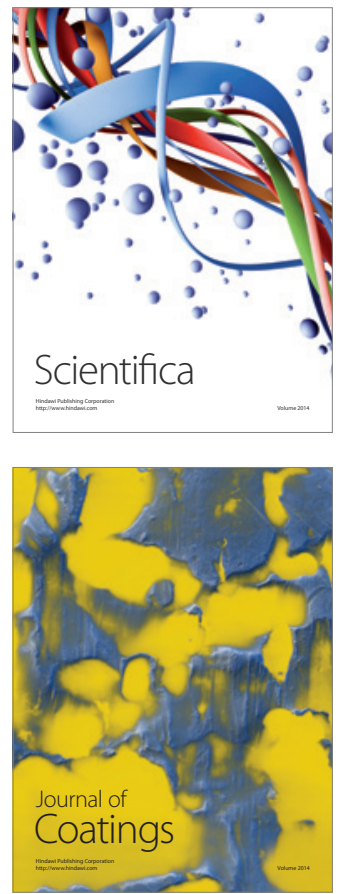
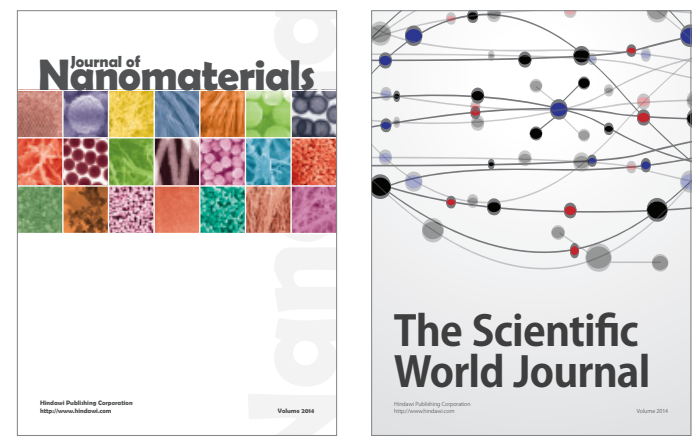

The Scientific World Journal
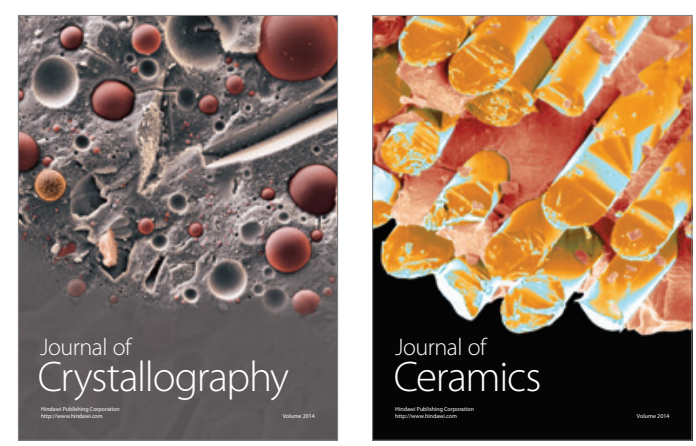
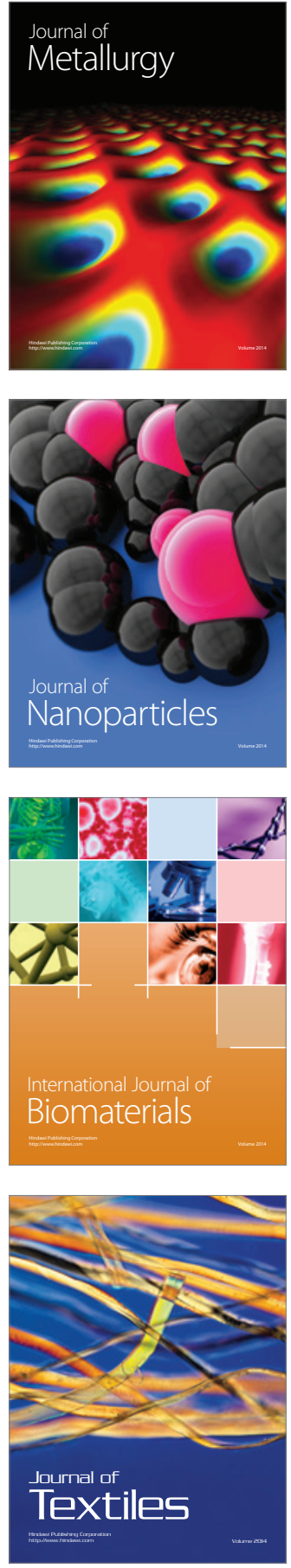